\title{
Comparison of HRV indices obtained from ECG and SCG signals from CEBS database
}

\author{
Szymon Siecinski ${ }^{1}$, Ewaryst J. Tkacz ${ }^{1,2^{*}}$ and Pawel S. Kostka ${ }^{1}$
}

\author{
*Correspondence: \\ etkacz@polsl.pl \\ 2 Katowice School \\ of Technology, 43 Rolna \\ Street, 40-055 Katowice, \\ Poland \\ Full list of author information \\ is available at the end of the \\ article
}

\begin{abstract}
Background: Heart rate variability (HRV) has become a useful tool of assessing the function of the heart and of the autonomic nervous system. Over the recent years, there has been interest in heart rate monitoring without electrodes. Seismocardiography (SCG) is a non-invasive technique of recording and analyzing vibrations generated by the heart using an accelerometer. In this study, we compare HRV indices obtained from SCG and ECG on signals from combined measurement of ECG, breathing and seismocardiogram (CEBS) database and determine the influence of heart beat detector on SCG signals.
\end{abstract}

Methods: We considered two heart beat detectors on SCG signals: reference detector using R waves from ECG signal to detect heart beats in SCG and a heart beat detector using only SCG signal. We performed HRV analysis and calculated time and frequency features.

Results: Beat detection performance of tested algorithm on all SCG signals is quite good on 85,954 beats ( $\mathrm{Se}=0.930, \mathrm{PPV}=0.934$ ) despite lower performance on noisy signals. Correlation between HRV indices was calculated as coefficient of determination $\left(R^{2}\right)$ to determine goodness of fit to linear model. The highest $R^{2}$ values were obtained for mean interbeat interval $\left(R^{2}=1.000\right.$ for reference algorithm, $R^{2}=0.9249$ in the worst case), $\mathrm{PSD}_{\mathrm{LF}}$ and PSD $\mathrm{HF}\left(R^{2}=1.000\right.$ for the best case, $R^{2}=0.9846$ for the worst case) and the lowest were obtained for PSDVLF ( $R^{2}=0.0009$ in the worst case). Using robust model improved achieved correlation between HRV indices obtained from ECG and SCG signals except the $R^{2}$ values of pNN50 values in signals p001-p020 and for all analyzed signals.

Conclusions: Calculated HRV indices derived from ECG and SCG are similar using two analyzed beat detectors, except SDNN, RMSSD, NN50, pNN50, and PSDVLF. Relationship of HRV indices derived from ECG and SCG was influenced by used beat detection method on SCG signal.

Keywords: Seismocardiography, Heart rate variability, HRV analysis

\section{Introduction}

Heart rate variability (HRV) is the physiological phenomenon of variation of time between heartbeats [1], which is caused by the activity of autonomic nervous system [2]. HRV has been frequently used in the analysis of physiological signals in different clinical and functional conditions [3, 4]. Low HRV is a risk factor for myocardial infarction, angina pectoris, and sudden cardiac death [5-8]. Other applications of HRV analysis 
include atrial fibrillation [9], brain stroke [10-14], sleep bruxism [15] diagnosis, and assessment the progress of rehabilitation of patients after ischemic brain stroke [16].

HRV is traditionally obtained from electrocardiogram (ECG) [17]. Over the recent years, there has been interest into non-invasive heart rate monitoring without using electrodes [18]. Seismocardiography (SCG) is a technique of recording and analyzing cardiac activity by measuring precordial acceleration. Recordings are taken using accelerometer on subjects in supine position [19]. In the past, SCG was mainly a tool for physiologists, due to the need of complex recording devices [20].

Technological improvements and miniaturization of accelerometers and the availability of low cost computational power have provided the reasons for reconsidering seismocardiography in clinical practice [21, 22]. Various applications have been proposed for SCG, including HRV analysis, detecting heart arrhythmia, and myocardial ischemia [22-24].

The feasibility of HRV analysis using SCG signals has been described earlier in papers [17, 18, 25-28]. Ramos-Castro et al. [18] and Landreani et al. [26, 27] showed that SCG signal acquired by smartphones can be used to perform HRV analysis. Laurin et al. [17] proved the validity of HRV indices obtained from SCG signal and Tadi et al. [25] study showed high correlation between HRV indices obtained from ECG and SCG.

The purpose of this study is to compare HRV indices obtained from SCG and ECG on signals from CEBS combined measurement of ECG, breathing, and seismocardiogram database and to determine the influence of heart beat detector on SCG signals. CEBS database is a multi-channel signal database available at PhysioNet.org [29-31]. A preliminary version of this work was presented in paper [28].

\section{Materials and methods}

Data set

CEBS database contains 60 multi-channel signals acquired on 20 healthy volunteers. Each recording consists of four channels with a sampling frequency of $5 \mathrm{kHz}$ : ECG (lead I and II), respiratory signal and SCG. Electrocardiogram (ECG) and respiratory signal were registered using Biopac MP36 data acquisition system. ECG (channel 1) was recorded with a bandwidth between 0.05 and $150 \mathrm{~Hz}$ and channel 4 (SCG) was recorded using the tri-axial accelerometer LIS334ALH by ST Microelectronics and $0.5-100 \mathrm{~Hz}$ bandwidth [29-31].

Subjects were asked to be awake and stay still in supine position on a bed during the measurement. After attaching the sensors, the basal state (before playing the music) was acquired for $5 \mathrm{~min}$ (recordings b001-b020). Then, the subjects started listening to music for 50 minutes (recordings m001-m020). Finally, the subjects were monitored for $5 \mathrm{~min}$ after the music ended (recordings p001-p020) [29, 31].

\section{ECG signal processing}

Several heart beat detectors have been proposed for ECG, which detect QRS complexes [32, 33]. In this study, we applied Pan-Tompkins algorithm [33] implemented by Wedekind [34] to detect R waves in ECG lead I. Pan-Tompkins algorithm consists of the following steps: band-pass filtering (to reduce noise, baseline wandering, muscle noise, etc.), differentiation, squaring of samples, moving average filtering, and correlation 
analysis [32, 33]. After preprocessing, amplitude thresholding is applied to identify $\mathrm{R}$ waves in the ECG signal. The interbeat intervals are calculated as differences between time of occurrence of successive $\mathrm{R}$ waves as in the following equation:

$$
t_{\mathrm{RR}, i}=t_{n}-t_{n-1},
$$

where $t_{\mathrm{RR}, i}$ is the $i$ th cardiac interval in ECG and $t_{n}$ denotes the occurrence of $n$th R wave.

\section{SCG signal processing}

Heart beat detection on seismocardiograms is based on nearly periodic appearance of fiducial points in SCG signal [35]. We chose Aortic valve opening (AO) wave which indicates the start of ventricular contraction and is usually visible as a single sharp wave [19].

In this study, we compare two beat detection algorithms: beat detection algorithm proposed by Tadi et al. in paper [25] used as a reference method of heart beat detection and the heart beat detector on SCG signals described in paper [24] described further as the tested beat detector.

\section{Reference beat detection algorithm}

Algorithm presented by Tadi et al. in 2015 [25] uses R waves as reference points and is based on the windowing method proposed in papers [36, 37]. The first step of the algorithm is applying a band-pass filter with cut-off frequencies of $4 \mathrm{~Hz}$ and $50 \mathrm{~Hz}$. Then, the SCG signal is smoothed using a moving average filter, whose window has the duration of 10-20 ms. The R waves in the ECG signal are localized using Pan-Tompkins algorithm and are the reference points. The location of $\mathrm{AO}$ wave of a cardiac cycle is determined as a maximum value of the SCG signal within a $90 \mathrm{~ms}$ window.

\section{Tested heart beat detector}

Beat detector proposed by Tadi et al. in 2016 [24] consists of the following steps: applying band-pass filtering to the signal (3rd order Butterworth filter with cut-off frequencies of $1 \mathrm{~Hz}$ and $45 \mathrm{~Hz}$ ), motion noise cancellation, Hilbert transform and applying band-pass filter with cut-off frequencies of $0.5 \mathrm{~Hz}$ and $3 \mathrm{~Hz}$ to obtain a waveform with the same periodicity as heart rate.

Motion noise detection consists of calculating signal power envelope, and thresholding. Signal power envelope is calculated from the SCG signal using root mean square operation and a sliding window with a length of $500 \mathrm{~ms}$. Signal parts, where the power envelope exceeds the threshold (twice the median value of signal power envelope), are classified as motion artifacts.

According to Tadi et al. [24], Hilbert transform improves the heart beat detection in SCG signals, because it facilitates the detection of the dominant peaks associated with heart beats. The envelope of the signal $s(t)$ can be obtained by applying the Hilbert transform defined in the following equation:

$$
\hat{s}(t)=\frac{1}{\pi} \int_{-\infty}^{+\infty} \frac{s(\tau)}{t-\tau} \mathrm{d} \tau .
$$

Hilbert transform yields a $90^{\circ}$ phase shift of $s(t)$ and thus we can calculate the magnitude of its envelope as in the following equation: 


$$
A(t)=\left|s_{a}(t)\right|=\sqrt{s^{2}(t)+\hat{s}^{2}(t)},
$$

where $s_{a}(t)$ is an analytic signal.

In the last step, we find local maxima of the magnitude of Hilbert envelope separated by at least $400 \mathrm{~ms}$. These maxima determine the positions of $\mathrm{AO}$ waves. The interbeat intervals in SCG are calculated as differences between timing points of successive AO waves as in the following equation:

$$
t_{\mathrm{AO}-\mathrm{AO}, i}=t_{n}-t_{n-1}
$$

where $t_{\mathrm{AO}, i}$ is the $i$ th cardiac interval in SCG and $t_{n}$ denotes the occurrence of $n$th AO wave.

\section{HRV analysis}

We calculated the mean interbeat interval (mean NN), the standard deviation of all interbeat intervals $(\mathrm{SDNN})$, the ratio of number of interbeat interval differences greater than $50 \mathrm{~ms}$ (NN50), the proportion calculated by dividing NN50 (pNN50) by the total number of interbeat intervals, the root mean square of differences (RMSSD) of successive RR intervals in accordance with current recommendations [2]. For frequency domain analysis, we used sampling frequency equal to $3 \mathrm{~Hz}$ and Hann window defined in the following equation:

$$
w(n)=\frac{1}{2}\left(1-\cos \left(2 \pi \frac{n}{N}\right)\right)
$$

where $N=L-1, L$ is the window length, and $0 \leq n \leq N$ [38].

The power of the low-frequency band $\left(\mathrm{PSD}_{\mathrm{LF}}\right.$ ) was computed in the band $0.04-0.15 \mathrm{~Hz}$, the power of very low-frequency band (PSD $\mathrm{VLF}$ ) was calculated for frequencies under $0.04 \mathrm{~Hz}$, and the power of the high frequency band $\left(\mathrm{PSD}_{\mathrm{HF}}\right)$ was computed in the band $0.15-0.4 \mathrm{~Hz}$. The LF/HF ratio was computed as the $\mathrm{PSD}_{\mathrm{LF}} / \mathrm{PSD}_{\mathrm{HF}}$ ratio.

\section{Results}

Due to the lack of annotations of recordings from CEBS database [39], the heart beats in SCG signal were annotated using the algorithm described in "Reference beat detection algorithm". Heart beats determined by this algorithm are treated as reference beats for SCG signal. Tested heart beat detector based on algorithm proposed in paper [24] was evaluated as the number of true positives (TP), false positives (FP), false negatives (FN), the number of beats, sensitivity, and positive predictive value (precision).

When the difference between position of reference $\mathrm{AO}$ wave and detected $\mathrm{AO}$ wave is within $180 \mathrm{~ms}$ margin, then this $\mathrm{AO}$ wave position is considered a true positive. False negative occurs when tested beat detector omits a true $\mathrm{AO}$ wave in reference annotation. False positive is determined for false detected $\mathrm{AO}$ wave.

Sensitivity (Se) is defined in the following equation:

$$
\mathrm{Se}=\frac{\mathrm{TP}}{\mathrm{TP}+\mathrm{FN}}
$$

and positive predictive value (PPV) is defined in the following equation:

$$
\mathrm{PPV}=\frac{\mathrm{TP}}{\mathrm{TP}+\mathrm{FP}}
$$


The number of beats is the sum of TP and FN. Table 1 presents beat detector performance measures on signals b001-b020. Table 2 presents beat detector performance measures on signals m001-m020 and Table 3 shows performance measures on signals p001-p020.

The best heart beat detection performance of tested algorithm within the analyzed series was achieved on signals m001-m020 (overall sensitivity of 0.939 and positive predictive value of 0.945 ) due to the lower number of false positive and false negative results. The worst overall performance was achieved on signals p001-p020 (overall sensitivity of 0.877 , precision of 0.857 ) and the performance of heart beat detection expressed as the overall sensitivity was 0.893 and for overall precision value of 0.896 .

Among the individual signals, the best results were achieved for recordings b002, b018, b019, p002, p016, p018, and p019 $(\mathrm{Se}=1.000, \mathrm{PPV}=1.000)$. The worst results were obtained for signal p006 $(\mathrm{Se}=0.176, \mathrm{PPV}=0.381)$, p003 $(\mathrm{Se}=0.273, \mathrm{PPV}=0.272)$, b003 (Se = 0.351, PPV = 0.395), and b005 $(\mathrm{Se}=0.385$, PPV =0.383) because of high levels of FP and FN which were caused by motion artifacts and the fact that the AO wave was not always the most prominent peak of the signal.

Mean and standard deviations of HRV indices obtained from interbeat intervals from ECG and SCG are presented in Table 4 for signals b001-b020, for signals m001-m020 in Table 5, in Table 6 for signals p001-p020, and in Table 7 for all analyzed signals.

Mean and standard deviation values of calculated indices are similar in each group of signals except SDNN, RMSSD, NN50, pNN50, and PSD VLF, where values achieved for tested algorithm are significantly greater. HRV indices mean and standard deviation are similar for 5-min signals (b001-b020 and p001-p020).

Table 1 Performance measures of tested heart beat detector on SCG signals b001-b020

\begin{tabular}{lrrrrrl}
\hline Signal & TP & FP & FN & Beats & Se & PPV \\
\hline b001 & 279 & 22 & 19 & 298 & 0.936 & 0.927 \\
b002 & 308 & 0 & 0 & 308 & 1.000 & 1.000 \\
b003 & 121 & 187 & 226 & 347 & 0.351 & 0.395 \\
b004 & 323 & 2 & 1 & 325 & 0.997 & 0.994 \\
b005 & 139 & 226 & 224 & 364 & 0.385 & 0.383 \\
b006 & 309 & 2 & 0 & 309 & 1.000 & 0.994 \\
b007 & 272 & 1 & 0 & 272 & 1.000 & 0.996 \\
b008 & 480 & 0 & 1 & 480 & 0.998 & 1.000 \\
b009 & 310 & 6 & 3 & 313 & 0.990 & 0.981 \\
b010 & 234 & 75 & 74 & 309 & 0.761 & 0.758 \\
b011 & 251 & 86 & 86 & 338 & 0.746 & 0.741 \\
b012 & 317 & 81 & 86 & 403 & 0.787 & 0.797 \\
b013 & 358 & 0 & 1 & 359 & 0.997 & 1.000 \\
b014 & 345 & 1 & 0 & 345 & 1.000 & 0.997 \\
b015 & 329 & 3 & 1 & 330 & 0.997 & 0.991 \\
b016 & 352 & 0 & 0 & 352 & 1.000 & 1.000 \\
b017 & 363 & 2 & 2 & 365 & 0.995 & 0.995 \\
b018 & 400 & 0 & 0 & 400 & 1.000 & 1.000 \\
b019 & 316 & 0 & 0 & 338 & 1.000 & 1.000 \\
b020 & 338 & 15 & 12 & 338 & 0.965 & 0.956 \\
Total & 6137 & 711 & 736 & 6873 & 0.893 & 0.896 \\
\hline
\end{tabular}


Table 2 Performance measures of tested heart beat detector on SCG signals m001-m020

\begin{tabular}{lrrrrrl}
\hline Signal & TP & FP & FN & Beats & Se & PPV \\
\hline$m 001$ & 3794 & 129 & 113 & 3907 & 0.971 & 0.967 \\
m002 & 3205 & 20 & 20 & 3225 & 0.994 & 0.994 \\
m003 & 2283 & 339 & 836 & 3119 & 0.732 & 0.871 \\
m004 & 3404 & 22 & 3 & 3407 & 0.999 & 0.994 \\
m005 & 1949 & 1656 & 1650 & 3599 & 0.542 & 0.541 \\
m006 & 3086 & 32 & 30 & 3116 & 0.990 & 0.990 \\
m007 & 2596 & 23 & 18 & 2614 & 0.993 & 0.991 \\
m008 & 5008 & 3 & 11 & 5019 & 0.998 & 0.999 \\
m009 & 2949 & 234 & 211 & 3160 & 0.933 & 0.926 \\
m010 & 2148 & 842 & 840 & 2988 & 0.719 & 0.718 \\
m011 & 3450 & 148 & 146 & 3596 & 0.959 & 0.959 \\
m012 & 3744 & 233 & 246 & 3990 & 0.938 & 0.941 \\
m013 & 3707 & 5 & 4 & 3711 & 0.999 & 0.999 \\
m014 & 3378 & 39 & 39 & 3417 & 0.989 & 0.989 \\
m015 & 3204 & 2 & 1 & 3205 & 1.000 & 0.999 \\
m016 & 3860 & 2 & 1 & 3861 & 1.000 & 0.999 \\
m017 & 3574 & 11 & 12 & 3586 & 0.997 & 0.997 \\
m018 & 4011 & 69 & 93 & 4104 & 0.977 & 0.983 \\
m019 & 3178 & 14 & 17 & 3195 & 0.995 & 0.996 \\
m020 & 3386 & 15 & 12 & 3398 & 0.996 & 0.996 \\
Total & 65,914 & 3838 & 4303 & 70,217 & 0.939 & 0.945 \\
\hline & & & & &
\end{tabular}

Table 3 Performance measures of tested heart beat detector on SCG signals p001-p020

\begin{tabular}{lrrrrrr}
\hline Signal & TP & FP & FN & Beats & Se & PPV \\
\hline p001 & 317 & 11 & 8 & 325 & 0.975 & 0.966 \\
p002 & 308 & 0 & 0 & 308 & 1.000 & 1.000 \\
p003 & 95 & 254 & 253 & 348 & 0.273 & 0.272 \\
p004 & 324 & 2 & 1 & 325 & 0.997 & 0.994 \\
p005 & 181 & 240 & 184 & 365 & 0.496 & 0.430 \\
p006 & 139 & 226 & 224 & 272 & 0.176 & 0.381 \\
p007 & 273 & 1 & 0 & 273 & 1.000 & 0.996 \\
p008 & 479 & 0 & 1 & 480 & 0.998 & 1.000 \\
p009 & 313 & 6 & 3 & 316 & 0.991 & 0.981 \\
p010 & 234 & 75 & 74 & 308 & 0.760 & 0.757 \\
p011 & 251 & 88 & 86 & 337 & 0.745 & 0.740 \\
p012 & 317 & 81 & 87 & 404 & 0.785 & 0.796 \\
p013 & 358 & 0 & 1 & 359 & 0.997 & 1.000 \\
p014 & 344 & 1 & 0 & 344 & 1.000 & 0.997 \\
p015 & 328 & 3 & 1 & 329 & 0.997 & 0.991 \\
p016 & 352 & 0 & 0 & 352 & 1.000 & 1.000 \\
p017 & 363 & 2 & 2 & 365 & 0.995 & 0.995 \\
p018 & 400 & 0 & 0 & 400 & 1.000 & 1.000 \\
p019 & 316 & 0 & 0 & 316 & 1.000 & 1.000 \\
p020 & 326 & 15 & 12 & 338 & 0.964 & 0.956 \\
Total & 6018 & 1005 & 937 & 6864 & 0.877 & 0.857 \\
\hline
\end{tabular}


Table 4 HRV indices derived from ECG lead I and SCG signal presented as mean and standard deviation (SD) on recordings b001-b020

\begin{tabular}{|c|c|c|c|c|c|c|}
\hline \multirow[t]{2}{*}{ HRV index } & \multicolumn{2}{|l|}{ ECG } & \multicolumn{2}{|c|}{ SCG (reference algorithm) } & \multicolumn{2}{|c|}{ SCG (tested algorithm) } \\
\hline & Mean & SD & Mean & SD & Mean & SD \\
\hline Mean NN [ms] & 880.6236 & 102.1249 & 880.6352 & 102.1375 & 877.9416 & 100.0651 \\
\hline SDNN [ms] & 55.3286 & 18.0816 & 58.8625 & 16.7121 & 92.5927 & 55.8870 \\
\hline RMSSD [ms] & 18.4905 & 21.1768 & 59.2054 & 20.4972 & 116.8905 & 100.4839 \\
\hline NN50 & 74.8000 & 46.9093 & 107.8000 & 42.1059 & 138.2500 & 84.8211 \\
\hline pNN50 & 0.2240 & 0.1408 & 0.3233 & 0.1319 & 0.4099 & 0.2501 \\
\hline$P S D_{L F}\left[\mathrm{~ms}^{2}\right]$ & $616,229.8079$ & $155,950.1659$ & $612,073.3519$ & $155,948.3816$ & $612,073.3519$ & $154,059.9771$ \\
\hline PSDVLF [ms2] & 2962.8256 & 2308.0362 & 3301.8585 & 2148.7678 & $10,018.8269$ & $15,211.3318$ \\
\hline $\mathrm{PSD}_{\mathrm{HF}}\left[\mathrm{ms}^{2}\right]$ & $616,714.3314$ & $155,958.1736$ & $616,713.8099$ & $155,953.0616$ & $612,867.8984$ & $153,989.0784$ \\
\hline $\mathrm{LF} / \mathrm{HF}$ & 0.9992 & 0.0006 & 0.9992 & 0.0006 & 0.9986 & 0.0022 \\
\hline
\end{tabular}

Table 5 HRV indices derived from ECG lead I and SCG signal presented as mean and standard deviation (SD) on recordings m001-m020

\begin{tabular}{|c|c|c|c|c|c|c|}
\hline \multirow[t]{2}{*}{ HRV index } & \multicolumn{2}{|l|}{ ECG } & \multicolumn{2}{|c|}{ SCG (reference algorithm) } & \multicolumn{2}{|c|}{ SCG (tested algorithm) } \\
\hline & Mean & SD & Mean & SD & Mean & SD \\
\hline Mean NN [ms] & 868.4482 & 107.7381 & 868.4494 & 107.7377 & 874.4701 & 108.1814 \\
\hline SDNN [ms] & 66.7848 & 27.6628 & 69.4776 & 27.2620 & 146.1656 & 259.5451 \\
\hline RMSSD [ms] & 54.2829 & 33.0621 & 63.9567 & 31.9611 & 177.9677 & 335.4112 \\
\hline NN50 & 699.5000 & 375.9997 & 1051.6500 & 480.1267 & 1223.8000 & 689.7342 \\
\hline pNN50 & 0.2068 & 0.1085 & 0.3098 & 0.1433 & 0.3621 & 0.2137 \\
\hline $\mathrm{PSD}_{\mathrm{LF}}\left[\mathrm{ms}^{2}\right]$ & $564,775.7879$ & $150,123.2398$ & $564,774.1763$ & $150,124.8666$ & $560,361.6889$ & $153,743.7541$ \\
\hline $\mathrm{PSD}_{\mathrm{VLF}}\left[\mathrm{ms}^{2}\right]$ & 2730.3091 & 1914.1473 & 3076.3276 & $150,124.8666$ & 7566.0048 & 8040.7530 \\
\hline $\mathrm{PSD}_{\mathrm{HF}}\left[\mathrm{ms}^{2}\right]$ & $565,323.1825$ & $150,225.7814$ & $565,321.7553$ & $150,228.3664$ & $561,047.5560$ & $153,853.2790$ \\
\hline $\mathrm{LF} / \mathrm{HF}$ & 0.9990 & 0.0005 & 0.9990 & 0.0005 & 0.9987 & 0.0008 \\
\hline
\end{tabular}

Table 6 HRV indices derived from ECG lead I and SCG signal presented as mean and standard deviation (SD) on recordings p001-p020

\begin{tabular}{|c|c|c|c|c|c|c|}
\hline \multirow[t]{2}{*}{ HRV index } & \multicolumn{2}{|l|}{ ECG } & \multicolumn{2}{|c|}{ SCG (reference algorithm) } & \multicolumn{2}{|c|}{ SCG (tested algorithm) } \\
\hline & Mean & SD & Mean & SD & Mean & SD \\
\hline Mean NN [ms] & 881.2197 & 102.3434 & 881.2311 & 102.3560 & 879.9381 & 100.3830 \\
\hline SDNN [ms] & 55.6498 & 17.7449 & 58.9799 & 16.5574 & 84.3181 & 34.9819 \\
\hline RMSSD [ms] & 48.4851 & 21.1828 & 58.4208 & 21.0497 & 103.2012 & 69.7998 \\
\hline NN50 & 168.1500 & 23.8311 & 167.8500 & 22.4365 & 132.8000 & 81.0884 \\
\hline pNN50 & 0.4899 & 0.0414 & 0.4892 & 0.0347 & 0.3911 & 0.2336 \\
\hline$P S D_{L F}\left[\mathrm{~ms}^{2}\right]$ & $616,967.1499$ & $156,145.0747$ & $616,968.3450$ & $156,144.2675$ & $616,229.7384$ & $153,878.4562$ \\
\hline PSDVLF $\left[\mathrm{ms}^{2}\right]$ & 2974.3376 & 2298.2310 & 3300.7693 & 2145.1549 & 7458.9407 & 7783.3257 \\
\hline $\mathrm{PSD}_{\mathrm{HF}}\left[\mathrm{ms}^{2}\right]$ & $617,450.7037$ & $156,152.3237$ & $617,453.1540$ & $156,148.1566$ & $616,747.0991$ & $153,885.5876$ \\
\hline $\mathrm{LF} / \mathrm{HF}$ & 0.9992 & 0.0006 & 0.9992 & 0.0006 & 0.9991 & 0.0006 \\
\hline
\end{tabular}

Tadi et al. [25] observed that HRV indices obtained from ECG and SCG have strong linear relationship. To examine the strength of linear correlation between HRV indices obtained from ECG and SCG, we used MATLAB Curve Fitting Tool to calculate 
Table 7 HRV indices derived from ECG lead I and SCG signal presented as mean and standard deviation (SD) on all analyzed recordings

\begin{tabular}{|c|c|c|c|c|c|c|}
\hline \multirow[t]{2}{*}{ HRV index } & \multicolumn{2}{|l|}{ ECG } & \multicolumn{2}{|c|}{ SCG (reference algorithm) } & \multicolumn{2}{|c|}{ SCG (tested algorithm) } \\
\hline & Mean & SD & Mean & SD & Mean & SD \\
\hline Mean NN [ms] & 876.7638 & 102.4935 & 876.7719 & 102.5018 & 874.4701 & 108.1814 \\
\hline SDNN [ms] & 59.2544 & 21.9539 & 62.4400 & 21.0417 & 146.1656 & 259.5451 \\
\hline RMSSD [ms] & 50.4195 & 25.4661 & 62.4400 & 24.7629 & 177.9677 & 335.4112 \\
\hline NN50 & 688.3000 & 754.5296 & 686.0833 & 750.9212 & 1223.800 & 689.7342 \\
\hline pNN50 & 0.4919 & 0.0366 & 0.4924 & 0.0353 & 0.3621 & 0.2137 \\
\hline$P S D_{L F}\left[m s^{2}\right]$ & $599,324.2486$ & $153,454.5209$ & $607,119.5110$ & $161,038.1290$ & $560,361.6889$ & $153,743.7541$ \\
\hline PSDVLF $\left[\mathrm{ms}^{2}\right]$ & 2889.1574 & 2146.9039 & 3226.3185 & 2003.5468 & 7566.0048 & 8040.7530 \\
\hline $\mathrm{PSD}_{\mathrm{HF}}\left[\mathrm{ms}^{2}\right]$ & $599,829.4059$ & $153,486.9967$ & $607,626.5532$ & $161,052.9574$ & $561,047.5560$ & $153,853.2790$ \\
\hline LF/HF & 0.9991 & 0.0006 & 0.9991 & 0.0006 & 0.9987 & 0.0008 \\
\hline
\end{tabular}

the goodness of fit to the 1st degree polynomial (linear) model. The goodness of fit is expressed as the coefficient of determination $R^{2}$.

Table 8 presents, Tables 9,10 , and 11 present correlation of determination $\left(R^{2}\right)$ calculated for linear model describing the relationship of HRV indices calculated from ECG and SCG on recordings b001-b020, m001-m020, p001-p020, and all recordings.

Table 8 Correlation between HRV indices obtained from ECG and SCG on recordings b001-b020

\begin{tabular}{lll}
\hline HRV index & $\boldsymbol{R}^{\mathbf{2}}$ (reference algorithm) & $\begin{array}{l}\boldsymbol{R}^{\mathbf{2}} \text { (tested } \\
\text { algorithm) }\end{array}$ \\
\hline Mean NN & 1.0000 & 0.9925 \\
SDNN & 0.9263 & 0.0533 \\
RMSSD & 0.5983 & 0.0546 \\
NN50 & 0.3566 & 0.0133 \\
PNN50 & 0.3854 & 0.0168 \\
PSDLF & 1.0000 & 0.9844 \\
PSDVLF & 0.9379 & 0.0416 \\
PSDHF & 1.0000 & 0.9862 \\
LF/HF & 0.9977 & 0.0360 \\
\hline
\end{tabular}

Table 9 Correlation between HRV indices obtained from ECG and SCG on recordings m001-m020

\begin{tabular}{|c|c|c|}
\hline HRV index & $R^{2}$ (reference algorithm) & $\begin{array}{l}R^{2} \text { (tested } \\
\text { algorithm) }\end{array}$ \\
\hline Mean NN & 1.0000 & 0.9249 \\
\hline SDNN & 0.9263 & 0.5507 \\
\hline RMSSD & 0.5983 & 0.4898 \\
\hline NN50 & 0.1791 & 0.1166 \\
\hline pNN50 & 0.2137 & 0.1580 \\
\hline$P S D_{L F}$ & 1.0000 & 0.9846 \\
\hline PSDVLF & 0.8967 & 0.0009 \\
\hline $\mathrm{PSD}_{\mathrm{HF}}$ & 1.0000 & 0.9846 \\
\hline $\mathrm{LF} / \mathrm{HF}$ & 0.9996 & 0.4296 \\
\hline
\end{tabular}


Table 10 Correlation between HRV indices obtained from ECG and SCG on recordings p001-p020

\begin{tabular}{lll}
\hline HRV index & $\boldsymbol{R}^{\mathbf{2}}$ (reference algorithm) & $\begin{array}{l}\boldsymbol{R}^{\mathbf{2}} \text { (tested } \\
\text { algorithm) }\end{array}$ \\
\hline Mean NN & 1.0000 & 0.9984 \\
SDNN & 0.9232 & 0.0043 \\
RMSSD & 0.9536 & 0.0176 \\
NN50 & 0.8957 & 0.0504 \\
pNN50 & 0.6782 & 0.0684 \\
PSD & 1.0000 & 0.9980 \\
PSDVLF & 1.0000 & 0.0094 \\
PSDHF & 1.0000 & 0.9980 \\
LF/HF & 0.9976 & 0.9629
\end{tabular}

Table 11 Correlation between HRV indices obtained from ECG and SCG on all recordings

\begin{tabular}{llc}
\hline HRV index & $\boldsymbol{R}^{\mathbf{2}}$ (reference algorithm) & $\begin{array}{l}\boldsymbol{R}^{\mathbf{2}} \text { (tested } \\
\text { algorithm) }\end{array}$ \\
\hline Mean NN & 1.0000 & 0.9681 \\
SDNN & 0.9232 & 0.2738 \\
RMSSD & 0.6092 & 0.2047 \\
NN50 & 0.3949 & 0.5800 \\
PNN50 & 0.4410 & 0.0617 \\
PSD & 1.0000 & 0.9889 \\
PSDVLF & 0.9390 & 0.0132 \\
PSDHF & 1.0000 & 0.9895 \\
LF/HF & 0.9976 & 0.1326
\end{tabular}

Figures 1 and 2 present linear model describing the relationship between mean $\mathrm{NN}$ calculated from ECG and SCG, and Figs. 3 and 4 present linear model of pNN50 derived from ECG and SCG.

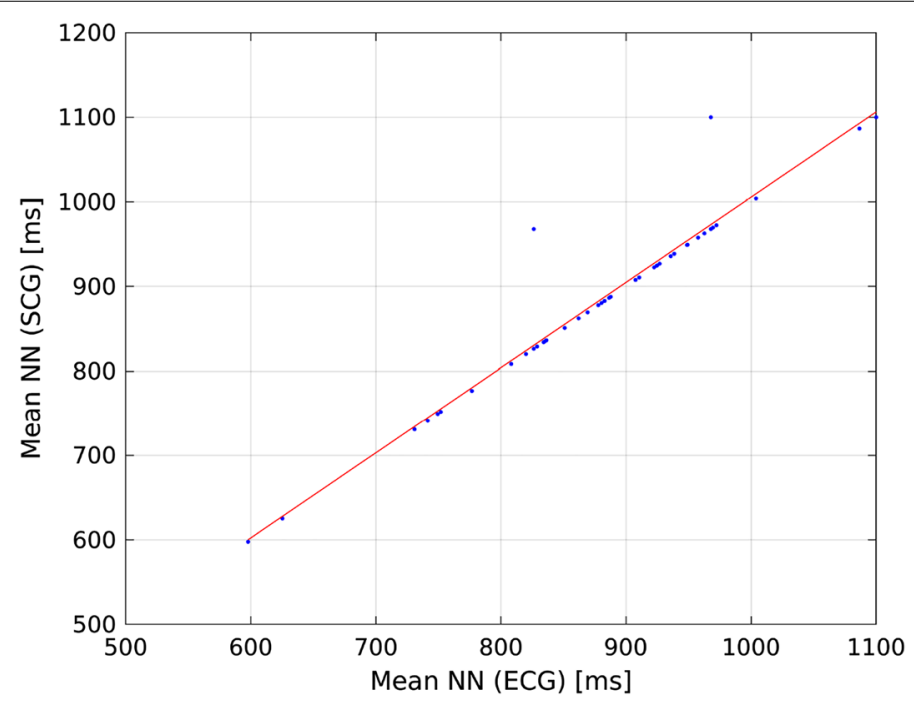

Fig. 1 Linear model describing the correlation between mean NN derived from SCG and mean NN calculated from SCG using reference algorithm 


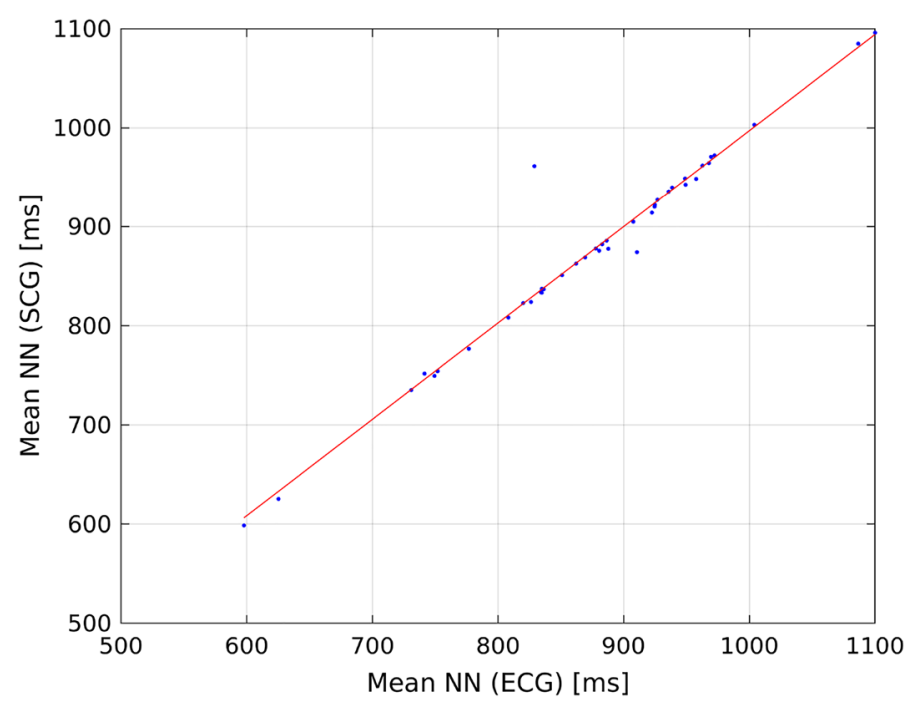

Fig. 2 Linear model describing the correlation between mean NN derived from SCG and mean NN calculated from SCG using tested algorithm

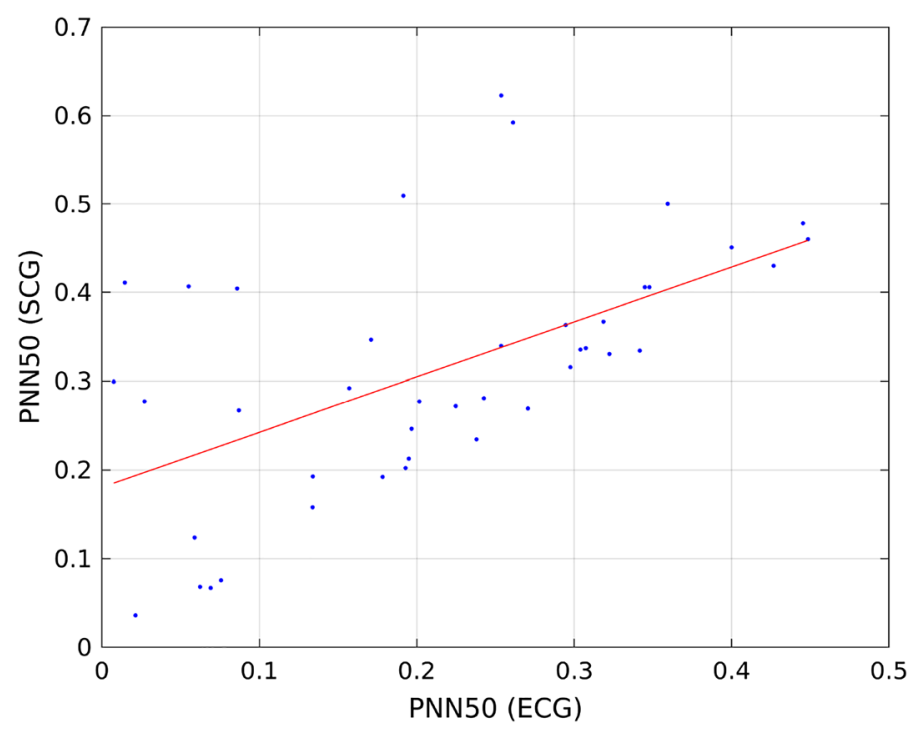

Fig. 3 Linear model describing the correlation between pNN50 derived from SCG and pNN50 calculated from SCG using reference algorithm

$R^{2}$ values calculated for linear fit between HRV indices derived from ECG and reference SCG beats indicate strong linear relationship except for NN50 and pNN50 in all signal groups except signals p001-p020 for NN50. When using tested heart beat detector, obtained $R^{2}$ values are lower for all recording groups. Mean NN, PSD $\mathrm{LF}_{\mathrm{LF}}$ and $\mathrm{PSD}_{\mathrm{HF}}$ have the maximum value of $R^{2}$ for each group of recordings. The weakest correlation was observed for SDNN, RMSSD, PNN50, and LF/HF for all groups of recordings, except LF/HF for recordings p001-p020. 


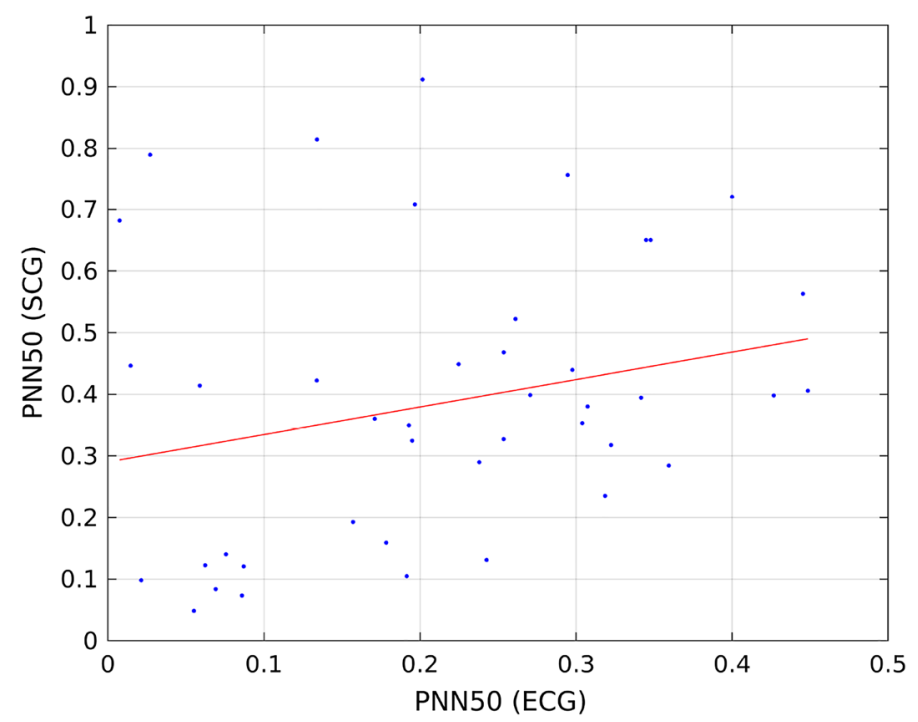

Fig. 4 Linear model describing the correlation between pNN50 derived from SCG and pNN50 calculated from SCG using tested algorithm

Table 12 Correlation between HRV indices obtained from ECG and SCG on recordings b001-b020 using robust linear model

\begin{tabular}{lll}
\hline HRV index & $\boldsymbol{R}^{\mathbf{2} \text { value }}$ & $\begin{array}{l}\text { Robust } \\
\text { model fit } \\
\text { method }\end{array}$ \\
\hline Mean NN & & LAR \\
SDNN & 0.9987 & LAR \\
RMSSD & 0.8422 & LSq \\
NN50 & 0.8424 & LAR \\
PNN50 & 0.8356 & LAR \\
PSDLF & 0.8271 & LSq \\
PSDVLF & 0.9977 & LAR \\
PSDHF & 0.8403 & LSq \\
LF/HF & 0.9977 & LAR
\end{tabular}

Despite the similarities of mean and standard deviation of analyzed HRV indices among the analyzed signals (heart beats obtained from ECG lead I, reference SCG beats and SCG with heart beats obtained using tested algorithm), there are significant differences in correlation between HRV indices between ECG and SCG signals. These discrepancies show that the correlation between HRV indices obtained from ECG and SCG depends on the quality of heart beat detection on SCG signals. To reduce the influence of the outliers in the model which are shown in Figs. 2 and 4, we applied robust model fitting using least absolute residual (LAR) method described further in [40] and least squares method (LSq). Then, the maximum value of $R^{2}$ coefficient was chosen as a result of robust model fitting. $R^{2}$ coefficients for recordings b001-b020 are shown in Table 12, for recordings m001-m020 are presented in Table 13 and $R^{2}$ values for recordings p001-p020 are shown in Table 14 . Table 15 presents the $R^{2}$ values for all analyzed recordings. 
Table 13 Correlation between HRV indices obtained from ECG and SCG on recordings m001-m020 using robust linear model

\begin{tabular}{lcc}
\hline HRV index & $\boldsymbol{R}^{\mathbf{2} \text { value }}$ & $\begin{array}{l}\text { Robust } \\
\text { model fit } \\
\text { method }\end{array}$ \\
\hline Mean NN & & LAR \\
SDNN & 0.9875 & LAR \\
RMSSD & 0.9251 & $\mathrm{LSq}$ \\
NN50 & 0.8424 & $\mathrm{LSq}$ \\
PNN50 & 0.2114 & $\mathrm{LSq}$ \\
PSD & 0.3109 & $\mathrm{LSq}$ \\
PSDVLF & 0.9974 & $\mathrm{LAR}$ \\
PSDHF & 0.8355 & $\mathrm{LSq}$ \\
LF/HF & 0.9974 & $\mathrm{LAR}$ \\
\hline
\end{tabular}

Table 14 Correlation between HRV indices obtained from ECG and SCG on recordings p001-p020 using robust linear model

\begin{tabular}{lcc}
\hline HRV index & $\boldsymbol{R}^{\mathbf{2}}$ value & $\begin{array}{l}\text { Robust } \\
\text { model fit } \\
\text { method }\end{array}$ \\
\hline Mean NN & & LSq \\
SDNN & 0.9984 & $\mathrm{LAR}$ \\
RMSSD & 0.8354 & $\mathrm{LSq}$ \\
NN50 & 0.1436 & $\mathrm{LAR}$ \\
PNN50 & 0.8417 & $\mathrm{LAR}$ \\
PSD & -0.0893 & $\mathrm{LSq}$ \\
PSDVLF & 0.9997 & $\mathrm{LAR}$ \\
PSDHF & 0.8349 & $\mathrm{LSq}$ \\
LF/HF & 0.9997 & $\mathrm{LAR}$ \\
\hline
\end{tabular}

Table 15 Correlation between HRV indices obtained from ECG and SCG on all recordings using robust linear model

\begin{tabular}{lcc}
\hline HRV index & $\boldsymbol{R}^{\mathbf{2} \text { value }}$ & $\begin{array}{l}\text { Robust } \\
\text { model fit } \\
\text { method }\end{array}$ \\
\hline Mean NN & & $\mathrm{LAR}$ \\
SDNN & 0.9970 & $\mathrm{LAR}$ \\
RMSSD & 0.9398 & $\mathrm{LSq}$ \\
NN50 & 0.9377 & $\mathrm{LAR}$ \\
PNN50 & 0.9374 & $\mathrm{LAR}$ \\
PSD $\mathrm{LF}$ & -0.0199 & $\mathrm{LAR}$ \\
PSDVLF & 0.9951 & $\mathrm{LAR}$ \\
PSDHF & 0.9390 & $\mathrm{LAR}$ \\
LF/HF & 0.9950 & $\mathrm{LAR}$ \\
\hline
\end{tabular}

Using robust model fitting improves the correlation between HRV indices obtained from ECG and SCG except for $p$ series and all analyzed signals due to the large number of outliers found in linear model. 


\section{Conclusion}

In this study, we presented the feasibility of HRV analysis using SCG signal and compared results obtained from ECG and SCG heart beats. Mean interbeat interval (Mean $\mathrm{NN}$ ), $\mathrm{PSD}_{\mathrm{LF}}$ and $\mathrm{PSD}_{\mathrm{HF}}$ are most robust to SCG signal noise and have the strongest linear correlation. HRV indices obtained from heart beat intervals using two different beat detectors on SCG signals are similar except SDNN, RMSSD, NN50, pNN50, and PSD $\mathrm{VLF}$, which are induced by noise in SCG signals and the limitations of tested beat detector. Using robust fitting to the linear model improves the correlation between HRV indices obtained from ECG and SCG signals [28] (except the $R^{2}$ values of pNN50 values in $p$ series and for all analyzed signals) and indicates the need to design a reliable heart beat detector which works on SCG signals.

Beat detection performance of tested algorithm on all SCG signals is quite good on 85,954 beats $(\mathrm{Se}=0.930$, PPV $=0.934)$ despite lower performance on noisy signals. Sensitivity and PPV on signals b001-b020 ( $\mathrm{Se}=0.893$, PPV $=0.896)$ is lower than reported in paper [28] $(\mathrm{Se}=0.995, \mathrm{PPV}=0.991)$ and Rivero et al. paper [41] $(\mathrm{Se}=0.99$, PPV $=0.97$ ). Tested algorithm has lower performance on SCG signals m001-m020 than reported in Li et al. [39] paper $(\mathrm{Se}=0.9933, \mathrm{PPV}=0.9941)$ due to the fact that tested algorithm based on the beat detector proposed by Tadi et al. [24] was susceptible to noise which strongly worsens the performance of heart beat detection. Other causes of worse performance include the occurrence of $\mathrm{AO}$ waves which are not the most prominent peaks and the fact that the heart beats on SCG signals detected as the nearest local maximum after the occurrence of the R wave in ECG signal may not occur within $90 \mathrm{~ms}$.

Strong linear relationship between most HRV indices obtained from ECG and SCG signals, especially between indices derived from ECG and reference beat detector on SCG signals, indicates the reliability of using SCG-derived interbeat intervals for HRV analysis $[18,25,28]$. Lower coefficients of determination between HRV indices obtained from ECG signal and beats detected on SCG signal using tested algorithm are caused by the noise found in analyzed signals. The possibility of recording and processing cardiac vibrations using one device broadens the scope of applicability of SCG [18, 25]. HRV analysis on SCG signal performed on smartphones may be used in mental stress assessment [27] or atrial fibrillation detection [42]. In future works, we will investigate the influence of other SCG beat detection algorithms on HRV indices.

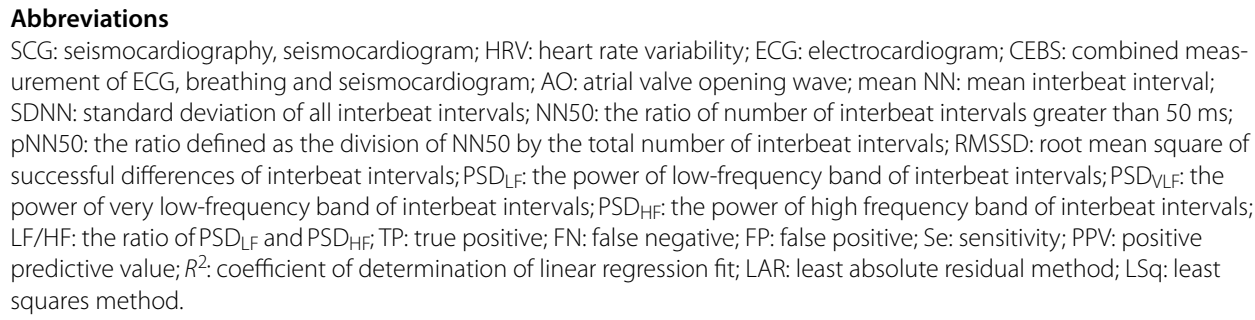


Availability of data and materials

The datasets used and/or analyzed during the current study are available from the corresponding author on reasonable request.

Ethics approval and consent to participate

Not applicable.

\section{Consent of publication}

Not applicable.

\section{Competing interests}

The authors declare that they have no competing interests.

\section{Author details}

${ }^{1}$ Department of Biosensors and Biomedical Signal Processing, Faculty of Biomedical Engineering, Silesian University of Technology, 40 Roosevelt's Street, 41-800 Zabrze, Poland. ${ }^{2}$ Katowice School of Technology, 43 Rolna Street, 40-055 Katowice, Poland.

Received: 21 March 2019 Accepted: 21 May 2019

Published online: 01 June 2019

\section{References}

1. Saykrs B. Analysis of heart rate variability. Ergonomics. 1973;16(1):17-32. https://doi.org/10.1080/001401373089244 79.

2. Task Force of the European Society of Cardiology the North American Society of Pacing Electrophysiology: Heart rate variability. Standards of measurement, physiological interpretation, and clinical use. Circulation. 1996;93:104365. https://doi.org/10.1161/01.CIR.93.5.1043.

3. Montano N, Porta A, Cogliati C, Costantino G, Tobaldini E, Casali KR, lellamo F. Heart rate variability explored in the frequency domain: A tool to investigate the link between heart and behavior. Neurosci Biobehav Rev. 2009;33(2): 71-80. https://doi.org/10.1016/j.neubiorev.2008.07.006. The Inevitable Link between Heart and Behavior: New Insights from Biomedical Research and Implications for Clinical Practice

4. Pumprla J, Howorka K, Groves D, Chester M, Nolan J. Functional assessment of heart rate variability: physiological basis and practical applications. Int J Cardiol. 2002;84(1):1-14. https://doi.org/10.1016/S0167-5273(02)00057-8.

5. Ho KKL, Moody GB, Peng C-K, Mietus JE, Larson MG, Levy D, Goldberger AL. Predicting survival in heart failure case and control subjects by use of fully automated methods for deriving nonlinear and conventional indices of heart rate dynamics. Circulation. 1997;96:842-8. https://doi.org/10.1161/01.CIR.96.3.842.

6. Algra A, Tijssen J. Heart rate variability from 24-hour electrocardiography and the 2-year risk for sudden death. Circulation. 1993;88:180-5. https://doi.org/10.1161/01.CIR.88.1.180.

7. Tsuji H, Venditti F, Manders E, Evans J, Larson M, Feldman C, Levy D. Reduced heart rate variability and mortality risk in an elderly cohort. The framingham heart study. Circulation. 1994;90:878-83.

8. Tsuji H, Larson M, Venditti F, Manders E, Feldman C, Levy D. Impact of reduced heart rate variability on risk for cardiac events. The framlingam heart study. Circulation. 1996;94(11):2850-5.

9. Barauskiene V, Rumbinaite E, Karuzas A, Martinkute E, Puodziukynas A. Importance of heart rate variability in patients with atrial fibrillation. J Cardiol Clin Res. 2016;4(6):1080.

10. Kawahara E, Ikeda S, Miyahara Y, Kohno S. Role of autonomic nervous dysfunction in electrocardio-graphic abnormalities and cardiac injury in patients with acute subarachnoid hemorrhage. Circ J. 2003;67(9):753-6. https://doi. org/10.1253/circj.67.753.

11. Watanabe N, Imai Y, Nagai K, Tsuji I, Satoh H, Sakuma M, Sakuma H, Kato J, Onodera-Kikuchi N, Yamada M, Fumiaki Abe F, Hisamichi S, Abe K. Nocturnal blood pressure and silent cerebrovascular lesions in elderly japanese. Stroke. 1996;27(6):1319-27. https://doi.org/10.1161/01.STR.27.8.1319.

12. Korpelainen J, Sotaniemi K, Tolonen U, Myllylä V. Cardiovascular autonomic reflexes in brain infarction. Stroke. 1994;25(4):787-92. https://doi.org/10.1161/01.STR.25.4.787.

13. Korpelainen J, Sotaniemi K, Tolonen U, Myllylä V. Abnormal heart rate variability as a manifestation of autonomic dysfunction in hemispheric brain infarction. Stroke. 1996;27(11):2059-63. https://doi.org/10.1161/01.STR.27.11.2059.

14. Korpelainen JT, Sotaniemi KA, Mäkikallio A, Huikuri HV, Myllylä VV. Dynamic behavior of heart rate in ischemic stroke. Stroke. 1999:30(5):1008-13. https://doi.org/10.1161/01.STR.30.5.1008.

15. Kostka PS, Tkacz EJ. Multi-sources data analysis with sympatho-vagal balance estimation toward early bruxism episodes detection. In: 2015 37th Annual international conference of the IEEE engineering in medicine and biology society (EMBC); 2015. p. 6010-3. https://doi.org/10.1109/EMBC.2015.7319761.

16. Tkacz E, Budzianowski Z, Oleksy W. The higher-order spectra as a tool for assessing the progress in rehabilitation of patients after ischemic brain stroke. In: Rocha Á, Guarda T, editors. Proceedings of the international conference on information technology \& systems (ICITS 2018). Cham: Springer; 2018. p. 874-82. https://doi.org/10.1007/978-3-31973450-7_83.

17. Laurin A, Blaber A, Tavakolian K. Seismocardiograms return valid heart rate variability indices. In: Computing in cardiology 2013; 2013. p. 413-6. https://ieeexplore.ieee.org/document/6713401.

18. Ramos-Castro J, Moreno J, Miranda-Vidal H, García-González MA, Fernández-Chimeno M, Rodas G, Capdevila L. Heart rate variability analysis using a seismocardiogram signal. In: 2012 annual international conference of the IEEE engineering in medicine and biology society; 2012. p. 5642-5. https://doi.org/10.1109/EMBC.2012.6347274. 
19. Zanetti JM, Salerno DM. Seismocardiography: a technique for recording precordial acceleration. In: [1991] computer-based medical systems. In: Proceedings of the fourth annual IEEE symposium; 1991. p. 4-9. https://doi. org/10.1109/CBMS.1991.128936.

20. Castiglioni P, Faini A, Parati G, Rienzo MD. Wearable seismocardiography. In: 2007 29th annual international conference of the IEEE engineering in medicine and biology society; 2007. p. 3954-7. https://doi.org/10.1109/IEMBS .2007.4353199.

21. Castiglioni P, Meriggi P, Rizzo F, Vaini E, Faini A, Parati G, Merati G, Rienzo MD. Cardiac sounds from a wearable device for sternal seismocardiography. In: 2011 annual international conference of the IEEE engineering in medicine and biology society; 2011. p. 4283-6. https://doi.org/10.1109/IEMBS.2011.6091063

22. Zanetti JM, Tavakolian K. Seismocardiography: past, present and future. In: 2013 35th annual international conference of the IEEE engineering in medicine and biology society (EMBC); 2013. p. 7004-7. https://doi.org/10.1109/ EMBC.2013.6611170

23. Inan OT, Migeotte PF, Park KS, Etemadi M, Tavakolian K, Casanella R, Zanetti J, Tank J, Funtova I, Prisk GK, Rienzo MD. Ballistocardiography and seismocardiography: a review of recent advances. IEEE J Biomed Health Inform. 2015;19(4):1414-27. https://doi.org/10.1109/JBHI.2014.2361732.

24. Tadi MJ, Lehtonen E, Hurnanen T, Koskinen J, Eriksson J, Pänkäälä M, Teräs M, Koivisto T. A real-time approach for heart rate monitoring using a Hilbert transform in seismocardiograms. Physiol Measure. 2016;37(11):1885-909. https ://doi.org/10.1088/0967-3334/37/11/1885.

25. Tadi MJ, Lehtonen E, Koivisto T, Pänkäälä M, Paasio A, Teräs M. Seismocardiography: Toward heart rate variability (HRV) estimation. In: Proceedings of the 2015 IEEE international symposium on medical measurements and applications (MeMeA); 2015. p. 261-6. https://doi.org/10.1109/MeMeA.2015.7145210.

26. Landreani F, Martin-Yebra A, Casellato C, Frigo C, Pavan E, Migeotte PF, Caiani EG. Beat-to-beat heart rate detection by smartphone's accelerometers: Validation with ecg. In: 2016 38th annual international conference of the IEEE engineering in medicine and biology society (EMBC); 2016. p. 525-8. https://doi.org/10.1109/EMBC.2016.7590755.

27. Landreani F, Morri M, Martin-Yebra A, Casellato C, Pavan E, Frigo C, Caiani EG. Ultra-short-term heart rate variability analysis on accelerometric signals from mobile phone. In: $2017 \mathrm{E}$-Health and bioengineering conference (EHB); 2017. p. 241-4. https://doi.org/10.1109/EHB.2017.7995406.

28. Siecinski S, Kostka PS, Tkacz EJ. Heart rate variability analysis on CEBS database signals. In: 2018 40th annual international conference of the IEEE engineering in medicine and biology society; 2018. p. 5697-700. https://doi. org/10.1109/EMBC.2018.8513551.

29. García-González MA, Argelagós-Palau A, Fernández-Chimeno M, Ramos-Castro J. A comparison of heartbeat detectors for the seismocardiogram. In: Computing in cardiology 2013; 2013. p. 461-4. https://ieeexplore.ieee.org/docum ent/6713413.

30. García-González MA, Argelagós A, Fernández-Chimeno M, Ramos-Castro J. Differences in QRS locations due to ECG lead: relationship with breathing. In: Roa Romero LM, editors. XIII Mediterranean conference on medical and biological engineering and computing 2013. Cham: Springer; 2014. p. 962-4. https://doi.org/10.1007/978-3-319-00846 -2 238.

31. Goldberger AL, Amaral LAN, Glass L, Hausdorff JM, Ivanov PC, Mark RG, Mietus JE, Moody GB, Peng C-K, Stanley HE. PhysioBank, PhysioToolkit, and PhysioNet: components of a new research resource for complex physiologic signals. Circulation. 2000; 101(23):215-220. Circulation Electronic Pages: http://circ.ahajournals.org/content/101/23/e215. full. https://doi.org/10.1161/01.CIR.101.23.e215.

32. Hamilton PS, Tompkins WJ. Quantitative investigation of qrs detection rules using the mit/bih arrhythmia database. IEEE Trans Biomed Eng BME. 1986;33(12):1157-65. https://doi.org/10.1109/TBME.1986.325695.

33. Pan J.Tompkins WJ. A real-time QRS detection algorithm. IEEE Trans Biomed Eng BME. 1985:32(3):230-6.

34. Wedekind D. Qrsdetector. TU Dresden, Institute for Biomedical Engineering, Biosignal Processing Group. https:// github.com/danielwedekind/arsdetector. Accessed 3 Feb 2018.

35. Zanetti JM, Poliac MO, Crow RS. Seismocardiography: waveform identification and noise analysis. In: [1991] Proceedings computers in cardiology; 1991. p. 49-52. https://doi.org/10.1109/CIC.1991.169042.

36. Pandia K, Inan OT, Kovacs GTA, Giovangrandi L. Extracting respiratory information from seismocardiogram signals acquired on the chest using a miniature accelerometer. Physiol Measure. 2012;33(10):1643-60. https://doi. org/10.1088/0967-3334/33/10/1643

37. Tadi MJ, Koivisto T, Pänkäälä M, Paasio A. Accelerometer-based method for extracting respiratory and cardiac gating information for dual gating during nuclear medicine imaging. Int J Biomed Imaging. 2014;2014(690124):1-11. https ://doi.org/10.1155/2014/690124.

38. Oppenheim AV, Schafer RW, Buck JR. Discrete-time signal processing. Upper Saddle River: Prentice Hall Press; 1999.

39. Li Y, Tang X, Xu Z. An approach of heartbeat segmentation in seismocardiogram by matched-filtering. In: 2015 7th international conference on intelligent human-machine systems and cybernetics, vol. 2; 2015. p. 47-51. https://doi. org/10.1109/IHMSC.2015.157.

40. Least absolute deviation regression. New York: Springer; 2008. p. 299-302. https://doi.org/10.1007/978-0-387-32833 $-1 \_225$.

41. Rivero Pouymiro I, Valdes Cordova E, Valdes Perez FE. Robust detection of AO and IM points in the seismocardiogram using CWT. IEEE Latin Am Trans. 2016;14(11):4468-73. https://doi.org/10.1109/TLA.2016.7795816.

42. Lahdenoja O, Hurnanen T, Iftikhar Z, Nieminen S, Knuutila T, Saraste A, Kiviniemi T, Vasankari T, Airaksinen J, Pänkäälä M, Koivisto T. Atrial fibrillation detection via accelerometer and gyroscope of a smartphone. IEEE J Biomed Health Inform. 2018;22(1):108-18. https://doi.org/10.1109/JBHI.2017.2688473.

\section{Publisher's Note}

Springer Nature remains neutral with regard to jurisdictional claims in published maps and institutional affiliations. 